\author{
GAME THEORY AND \\ MATHEMATICAL ECONOMICS \\ BANACH CENTER PUBLICATIONS, VOLUME 71 \\ INSTITUTE OF MATHEMATICS \\ POLISH ACADEMY OF SCIENCES \\ WARSZAWA 2006
}

\title{
SERIAL COST SHARING
}

\author{
ELENA YANOVSKAYA \\ Institute for Economics and Mathematics, Russian Academy of Sciences \\ Tchaikovsky st. 1, 191187 St. Petersburg, Russia \\ E-mail: eyanov@iatp20.spb.org
}

\begin{abstract}
A new axiomatization of the serial method for heterogeneous cost sharing problems is given. One of the axioms is common ordinality. It requires invariance of the method w.r.t. common ordinal transformations of individual utilities.
\end{abstract}

1. Introduction. The fair division of a joint cost (or a jointly produced output) among agents with different shares of input or output commodities is a central theme of the theory of cooperative games with transferable utility as well of public utility pricing, accounting, joint production of public or private goods etc.

The problem of rationing a single commodity according to a profile of claims (demands, liabilities) is the simplest example of fair division problems (see the surveys [6], [10]). Rationing problems have many interpretation e.g., taxation, bankruptcy, and inheritance.

Formally, a rationing problem is a triple $\langle N, T ; g\rangle$, where $N$ is a set of agents, $g \in \mathbb{R}_{+}^{N}$ is a vector of demands (claims), and $T \leq \sum_{i \in N} g_{i}$ is the total amount to be divided. A rationing method (rule) is a mapping associating with each rationing problem $\langle N, T ; g\rangle$ a vector of shares $x(T ; g) \in \mathbb{R}_{+}^{N}$ such that $\sum_{i \in N} x_{i}(T ; g)=T$.

The more general model where the values of a cost function $C: \mathbb{R}_{+}^{N} \rightarrow \mathbb{R}_{+}^{N}$ are to be shared may be considered either as a cost sharing or as an output-sharing one. In the cost sharing version each agent demands a different good, and the technology specifies the total cost $C\left(x_{1}, \ldots, x_{n}\right)$ to be divided. In the output sharing version each agent $i$ contributes the amount $x_{i}$ of his input. Then the total output $C\left(x_{1}, \ldots, x_{n}\right)$ should be divided among the participants. The function $C$ is considered here as a production function, so the goods

2000 Mathematics Subject Classification: Primary 91B32.

JEL Classification: D63, C71.

Key words and phrases: heterogeneous cost sharing problem, additive cost sharing method, serial method, path-generated method, common ordinality.

Research was supported by RFBR grant 04-06-80316.

The paper is in final form and no version of it will be published elsewhere. 
are identified with the agents. All information about the costs involved with bringing production up to a certain level is given by the cost function. Thus, the cost sharing rule must rely exclusively on the cost function and agents' demands.

Examples of cost sharing problems include sharing the cost of a network connecting dispersed users, where heterogeneity of demands comes from heterogeneity of space, or of a telecommunication network in which the users need different service. In an output problem various divisions of the firm can contribute heterogeneous inputs in a common project. At the same time the divisions may share a common service and another cost sharing problem how to pay for this service arises.

A heterogeneous cost sharing problem is a triple $\langle N, C ; q\rangle$, where $N$ is a finite set of agents, $C: \mathbb{R}_{+}^{N} \rightarrow \mathbb{R}_{+}$is a nondecreasing cost function such that $C(0)=0$, and $q \in \mathbb{R}_{+}^{N}$ is a profile of demands. When the set $N$ is fixed we shall denote the problem $\langle N, C, q\rangle$ simply by $\langle C, q\rangle$.

Note that the mathematical complexity of the last model is much more above the rationing one. For example, the binary cost sharing problem when $x_{i}$ (and also $q_{i}$ ) are equal to 0 or 1 for all $i \in N$ is equivalent to a cooperative game with transferable utilities $(N, v)$, where $v(S)=C\left(q_{S}, 0\right) \forall S \subset N, q_{S}$ is the projection of vector $q$ on the space $\mathbb{R}_{+}^{S}$.

If the function $C$ depends only on the sum of demands: $C(x)=C_{0}\left(x_{1}+\ldots+x_{n}\right)$, where $C_{0}: \mathbb{R}_{+} \rightarrow \mathbb{R}_{+}$is a non-decreasing function, then we have a homogeneous problem.

A solution of the problem $\langle N, C, q\rangle$ is a vector $y \in \mathbb{R}_{+}^{N}$ such that $\sum_{i \in N} y_{i}=C(q)$.

A cost sharing method (rule) is a mapping $\varphi$ associating with any problem $\langle N, C, q\rangle$ a solution $y=\varphi(N, C, q)$.

Many cost sharing rules have been defined and studied [6]. Three different methods have received the main attention in the literature. They are the Shapley-Shubik cost sharing method which is defined as the Shapley value of the TU game generated by the cost sharing problem, the Aumann-Shapley method [1] which generalizes the proportional method for homogeneous problems to the general case, and the serial method or the Friedman-Moulin method [3] which charges each agent by the integral of his marginal cost along the piecewise diagonal curve linking (in case of $q_{1}<q_{2}<\ldots<q_{n}$ ) the origin to $\left(q_{1}, \ldots, q_{1}\right)$, then to $\left(q_{1}, q_{2}, \ldots, q_{2}\right)$ etc. to $q=\left(q_{1}, q_{2} \ldots, q_{n}\right)$.

The axiomatizations of all the methods have been given in [3]. However, opposite to the first two methods, the axiomatization of the serial method is not enough compelling, because it uses an axiom placing a restriction on the method over the restricted class of cost functions with homogeneous goods.

In the paper a new axiom - Common Ordinality - is defined as a weakening of Ordinality introduced by Sprumont [8]. It requires that a change in a common ordinal scale of goods or agents' efforts should have no effect. This axiom is compelling when different goods or agents' efforts are genuinely comparable. The main result is the new axiomatization of the serial cost sharing method using the axiom Common Ordinality.

Section 2 briefly reviews the model and the known properties of cost sharing methods. The integral representation of all methods satisfying the Additivity and Dummy axioms due to Moulin-Friedman [3] is also presented. The axiomatic characterization of the serial cost sharing method is given in Section 3. 
2. Properties of cost sharing methods. The agents' cost shares $y_{i}$ should reflect responsibilities in generating the costs. A minimal requirement to that effect is that an agent who is not generating any cost should pay nothing. The Dummy axiom conveys just that idea.

Dummy (DUM). If the function $C$ does not depend on the $i$-th variable: $C(q)=$ $C\left(q_{N \backslash\{i\}}, 0\right)$, then $y_{i}=\varphi(N, C, q)=0$ for every $q \in \mathbb{R}_{+}^{N}$.

The additivity axiom is formulated as usual:

\section{Additivity (ADD).}

$$
\left.\varphi\left(N, C^{1}+C^{2}\right), q\right)=\varphi\left(N, C^{1}, q\right)+\varphi\left(N, C^{2}, q\right) \text { for all } N, C, q .
$$

The set of all methods satisfying Dummy and Additivity is denoted by $\mathfrak{F}(D U M, A D D)$. A similar notation will be used in the sequel for cost sharing methods satisfying other properties as well.

Demand Continuity (DCONT). The function $\varphi(N, C, q)$ is continuous in $q$.

Demand Monotonicity (DM). For any $C, q$

$$
\left.q_{i} \leq q_{i}^{\prime} \Longrightarrow \varphi_{i}(q ; C) \leq \varphi_{i}\left(q \| q_{i}^{\prime}\right) ; C\right)
$$

where the vector $q \| q_{i}^{\prime}$ is defined by

$$
\left(q \| q_{i}^{\prime}\right)_{j}= \begin{cases}q_{j}, & \text { if } j \neq i, \\ q_{i}^{\prime}, & \text { if } j=i .\end{cases}
$$

Symmetry (SYM). A cost sharing method $\varphi$ is symmetric if for any problem $\langle N, C, q\rangle$ with a cost function $C$ symmetric in the demands $i, j \in N$ the equality $q_{i}=q_{j}$ implies $\varphi_{i}(N, C, q)=\varphi_{j}(N, C, q)$.

Scale Invariance (SI). For any problem $\langle N, C, q\rangle$ and vector $a \in \mathbb{R}_{++}^{N} \varphi\left(N, C^{a}, q / a\right)=$ $\varphi(N, C, q)$, where $C^{a}(x)=C(a x), a x=\left(a_{i} x_{i}\right)_{i \in N}, q / a=\left(q_{i} / a_{i}\right)_{i \in N}$.

A path $\gamma$ to a point $q \in \mathbb{R}_{+}^{N}$ is a continuous nondecreasing curve connecting the origin 0 with $q$. Hence, there exists a parametric representation $\gamma(t, q), t \in[0, T]$ for every path $\gamma$ :

$$
x \in \gamma \Longleftrightarrow x_{i}=\gamma_{i}(t, q) \text { for all } i \in N \text { and some } t \in[0, T] .
$$

In representation (1) the functions $\gamma_{i}(q, t)$ are continuous and nondecreasing in $t$. From the representation it follows that $\gamma(q, 0)=0, \gamma(q, 1)=q$ for all $q \in \mathbb{R}_{+}^{N}$.

Denote by $\mathfrak{C}$ the set of all continuously differentiable cost functions. With any path $\gamma$ we can associate the cost sharing method $x^{\gamma}$ such that for any $C \in \mathfrak{C}$

$$
x_{i}^{\gamma}(C, q)=\int_{0}^{T} \partial_{i} C(\gamma(q, t)) d \gamma_{i}(q, t),
$$

where $\partial_{i} C$ denotes the $i$-th partial derivative of $C$.

Such path generated methods form the extreme points of the set $\mathfrak{F}(A D D, D U M)$.

The converse result was proved by Haimanko [4]:

TheOREM 1 (Haimanko (1998)). $\varphi(q, \cdot) \in \operatorname{ext} \mathfrak{F}(A D D, D U M)$ is an extreme point of the set $\mathfrak{F}(A D D, D U M)$ (for fixed $q$ ) if and only if there exists a path $\gamma(q, \cdot)$ such that $\varphi(q, \cdot)=x^{\gamma}(q, \cdot)$. In such a case the method $\varphi$ is called path-generated. 
Friedman and Moulin [3] proved the following representation theorem:

Theorem 2 (Friedman, Moulin (1999)). If $\varphi \in \mathfrak{F}(A D D, D U M)$ then there exists a measure $\nu$ on $\Gamma(q)$ such that

$$
\varphi(q, \cdot)=\int_{\Gamma(q)} x^{\gamma}(q, \cdot) d \nu(\gamma),
$$

where $\Gamma(q)$ is the set of all paths from 0 to $q$.

Theorems 1 and 2 establish one-to-one correspondence between the set of extreme points of $\mathfrak{F}(A D D, D U M)$ and the set of rationing methods. In fact, the locus of the vector of shares chosen by a rationing method when the total amount varies from 0 to the sum of the claims is the monotonic path $\gamma(t, q), t \in[0, T]$ connecting zero (at $t=0$ ) with the demand vector $q$ at $t=T$.

Y. Sprumont [8] introduced a property of the cost sharing methods known as Ordinality. This property formally reminds the ordinal measurability property of social welfare functions and requires robustness of cost sharing methods with respect to independent ordinal transformations of measuring scales. To define Ordinality we give some notation.

Let $N$ be a fixed finite set. Consider a list $\left(f_{1}, \ldots, f_{n}\right)$ of $n$ bijections from $\mathbb{R}_{+}$onto itself. For each cost function $C: \mathbb{R}_{+}^{N} \rightarrow \mathbb{R}_{+}$define another cost function $C^{f}: \mathbb{R}_{+}^{N} \rightarrow \mathbb{R}_{+}$ by

$$
C^{f}(q)=C(f(q)) \text {, where } f(q)=\left(f_{1}\left(q_{1}\right), \ldots, f_{n}\left(q_{n}\right)\right) .
$$

We call the problem $\left\langle C^{f}, f^{-1}(q)\right\rangle$ the ordinal transformation of the problem $\langle C, q\rangle$. Here $f^{-1}(q)=\left(f_{1}^{-1}\left(q_{1}\right), \ldots, f_{n}^{-1}\left(q_{n}\right)\right)$.

When we consider a special class of cost functions (e.g. continuous, differentiable etc.) we will suppose that the functions $f_{i}$ possess the same properties in order the ordinal transformations belong to the same class.

Two problems $\langle C, q\rangle$ and $\left\langle C^{\prime}, q^{\prime}\right\rangle$ are ordinally equivalent if there exists an ordinal transformation $f$ such that

$$
C^{\prime}=C^{f} \quad \text { and } \quad q=f\left(q^{\prime}\right) .
$$

Ordinality (ORD). Let $N$ be an arbitrary fixed set. If $\langle C, q\rangle$ and $\left\langle C^{\prime}, q^{\prime}\right\rangle$ are two ordinally equivalent problems, then $\varphi(C, q)=\varphi\left(C^{\prime}, q^{\prime}\right)$.

Evidently, Scale Invariance is a weakening of Ordinality. In fact, Scale Invariance is defined in the same way as Ordinality, but only for linear functions $f_{i}(x)=a_{i} x, a_{i}>0$.

It is easy to note that if all path-generated methods belonging to the "mixing set" $\Gamma(q)$ in representation formula (3) satisfy Ordinality then so does the method $\varphi$.

Each cost allocation problem $\langle N, C, q\rangle$ generates a TU (cost) cooperative game $\left\langle N, C_{q}\right\rangle$ by

$$
C_{q}(S)=C\left(q_{S}, 0_{N \backslash S}\right)
$$

The game $\left\langle N, C_{q}\right\rangle$ is called the stand-alone (cost) game.

Simplicity (SIM). The method $\varphi$ satisfies Simplicity if for any problem $\langle N, C, q\rangle$ the solution $\varphi(N, C, q)$ depends only on the values $C_{q}(S), S \subset N$. 
Evidently, Simplicity implies Ordinality, but not vice versa. In fact, Ordinality allows to use much more information than just "stand-alone cost data".

However, it turns out that among the methods from $\mathfrak{F}(A D D, D U M)$ Ordinality implies Simplicity: Sprumont and Wang [9] have proved that any cost sharing method $\varphi \in \mathfrak{F}(A D D, D U M, O R D)$ is a random order value.

A random order value is the method being a convex combination of the incremental methods. Each incremental method $\varphi^{\pi}$ is an extreme method from $\mathfrak{F}(A D D, D U M)$ and is defined by the marginal solution of the stand alone game corresponding to the permutation $\pi: N \rightarrow N$.

Definition of path-generated methods implies that the path defining the incremental method $\varphi^{\pi}$ is going through the following edges of the rectangle $[0, q]$ :

$$
\left[0,\left(q_{i_{1}}, 0\right)\right],\left[\left(q_{i_{1}}, 0\right),\left(q_{i_{1}}, q_{i_{2}} ; 0\right)\right], \ldots,\left[\left(q_{i_{1}}, \ldots, q_{i_{n-1}}, 0\right), q\right] .
$$

From the Sprumont and Wang theorem it follows that the class $\mathfrak{F}(A D D, D U M, S Y M$, $O R D)$ consists only of the Shapley value of the corresponding stand-alone game. This method is called the Shapley-Shubik method. First this fact was proven earlier by Sprumont [8]. Friedman and Moulin [3] have characterized the Shapley-Shubik method by ADD, DUM, SYM, Scale Invariance (SI) and Demand Monotonicity (DM). Thus, the Shapley-Shubik method has the compelling axiomatic characterizations.

However, another popular method from $\mathfrak{F}(A D D, D U M)$ - the serial method-has a less convincing characterization due also to Friedman and Moulin [3]. ${ }^{1}$ Let us give some definitions.

The next axiom describes a property of cost sharing methods for homogeneous problems:

Upper bound for homogeneous goods (UBH). $\varphi_{i}\left(q, C_{0}\right) \leq C_{0}\left(k q_{i}\right)$, where $k \leq n$ is the number of nondummy agents (goods).

When goods are homogeneous, the axiom requires that no agent should pay more than the cost of producing $n$ times his own demand. This bound protects the agent with a demand below the average from being charged too much: if marginal costs increase, the low demand agent should not be held responsible for the high marginal cost generated by demands larger than his own. Symmetrically, in the output sharing interpretation, UBH places a ceiling on the share awarded to an agent who contributes less than the average contribution.

The serial cost sharing method $s$ is given by the following formula:

$$
s_{i}(q, C)=\int_{0}^{q_{i}} \partial_{i} C((t e) \wedge q) d t
$$

where $e=(1, \ldots, 1),(a \wedge b)_{i}=\min \left\{a_{i}, b_{i}\right\}$. In other words, the serial method is a pathgenerated method whose paths correspond to the uniform gains rationing method.

\footnotetext{
${ }^{1}$ Recently Friedman [2] defined the consistency property of heterogeneous cost sharing methods satisified by the serial method. He obtained sufficient conditions for consistency cost sharing methods. With the help of consistency it would be possible to obtain another characterization of the serial method.
} 
Theorem 3 (Friedman, Moulin (1999)). The serial method is the unique method satisfying $A D D, D U M, D M$ and $U B H$.

In this theorem axiom UBH seems to be not compelling because it prescribes the property only for homogeneous problems, and it is not clear how the methods for heterogeneous and homogeneous problems should be connected. It would be more natural if the agents receive similar protections through some other properties mostly concerned with Independence and Monotonicity.

3. An axiomatization of the serial method. The main result of the paper is a new axiomatization of the serial method. Note that the serial method does not satisfy ordinality. In fact, independent bijections $f_{i}, i \in N$, participating in the definition of ordinal transformations of a problem $\langle C, q\rangle$ can change relative values of demands, e.g. there may arise the inequalities

$$
q_{i}<q_{j} \text { and } f_{i}\left(q_{i}\right)>f_{j}\left(q_{j}\right),
$$

but the serial method distinguishes the agents with greater and smaller demands. Thus, we weaken Ordinality replacing independent bijections $f_{i}, i \in N$ by identical ones: $f, \ldots, f: \mathbb{R}_{+} \rightarrow \mathbb{R}_{+}$(compare the Ordinal Measurability and Ordinal Full Comparability properties of social welfare functions).

Let $N$ be fixed. For each cost function $C: \mathbb{R}_{+}^{N} \rightarrow \mathbb{R}_{+}$define another cost function $C^{f}: \mathbb{R}_{+}^{N} \rightarrow \mathbb{R}_{+}$by

$$
C^{f}(q)=C(f(q)) \text {, where } f(q)=\left(f\left(q_{1}\right), \ldots, f\left(q_{n}\right)\right) .
$$

We call the problem $\left\langle C^{f}, f^{-1}(q)\right\rangle$ the coordinal transformation of the problem $\langle C, q\rangle$. Here $f^{-1}(q)=\left(f^{-1}\left(q_{1}\right), \ldots, f^{-1}\left(q_{n}\right)\right)$.

Common ordinality (COORD). Let $N$ be an arbitrary fixed set. If $\left\langle C^{f}, f^{-1}(q)\right\rangle$ is a coordinal transformation of $\langle C, q\rangle$ then $\varphi(C, q)=\varphi\left(C^{f}, f^{-1}(q)\right)$.

The last definition means that if a method satisfies COORD then every agent's utility is measured in a common ordinal scale. Evidently, ORD implies COORD. The problems $\langle C, q\rangle,\left\langle C^{\prime}, q^{\prime}\right\rangle$ which are coordinally equivalent preserve the order between the numbers $q_{i}, i \in N$, i.e. the correspondence "poor-rich" that is essential in the serial method.

Proposition 1. The serial method satisfies COORD.

Proof. Let $\langle C, q\rangle,\left\langle C^{f}, f^{-1}(q)\right\rangle$ be two coordinally equivalent problems. Then, by (4),

$$
\begin{aligned}
s_{i}\left(C^{f}, f^{-1}(q)\right) & =\int_{0}^{f^{-1}\left(q_{i}\right)} \partial_{i} C^{f}\left((t e) \wedge\left(f^{-1}\left(q_{i}\right)\right) d t\right. \\
& =\int_{0}^{f^{-1}\left(q_{i}\right)} \partial_{i} C((f(t) e) \wedge q) f^{\prime}(t) d t=\int_{0}^{q_{i}} C((\tau e) \wedge q) d \tau=s_{i}(C, q) .
\end{aligned}
$$

Thus, $s \in \mathfrak{F}(A D D, D U M, C O O R D)$. The next lemma describes the extreme points of the set $\mathfrak{F}(A D D, D U M, C O O R D)$.

LEMma 1. If a method $\varphi(q, \cdot)$ is an extreme point of the set $\mathfrak{F}(A D D, D U M, C O O R D)$, then the path $\gamma^{\varphi}(t, q), t \in[0, T]$, corresponding to $\varphi$, is piecewise linear. The $i$-th coordinate $\gamma^{\varphi}(t, q)$ of the end point of each segment of the path is equal either to zero or to 
$q_{j}, j \in N, q_{j} \leq q_{i}$ for arbitrary $i \in N$. On each segment $\alpha$ the coordinates $\gamma_{i}^{\varphi}(t, q), i \in S_{\alpha}$ are equally increasing for some $S_{\alpha} \subset N$, and other coordinates $\gamma_{i}(t, q)$ for $i \in N \backslash S_{\alpha}$ are constant (and equal to 0 or $q_{j}$ for some $j \in N$. such that $q_{j} \leq q_{k} \forall k \in S_{\alpha}$ ).

Proof. Let $\varphi(N, q, \cdot) \in \operatorname{ext} \mathfrak{F}(A D D, D U M, C O O R D)$. By Haimanko's theorem

$$
\varphi_{i}(N, q, C)=\int_{0}^{T} \partial_{i} C(\gamma(t, q)) d \gamma_{i}^{\varphi}(t, q),
$$

where $\gamma^{\varphi}(t, q)$ is the path generating the method $\varphi, \gamma^{\varphi}(0, q)=\mathbf{0}, \gamma^{\varphi}(T, q)=q$.

Consider the following possibilities for the path $\gamma^{\varphi}$ :

Case 1. $\gamma^{\varphi}$ is a strictly monotone path from 0 to $g$. Then it is possible to parametrize this path in such a way that $\gamma_{1}^{\varphi}(t, q)=t, t \in\left[0, q_{1}\right]$, and $\gamma_{i}^{\varphi}(t, q), i \neq 1$ are strictly increasing functions with the domain $\left[0, q_{1}\right]$. Then equality (5) can be rewritten as follows:

$$
\begin{aligned}
\varphi_{1}(N, C, q) & =\int_{0}^{q_{1}} \partial_{1} C\left(t, \gamma_{2}^{\varphi}(t, q), \ldots, \gamma_{n}^{\varphi}(t, q)\right) d t \\
\varphi_{i}(N, C, q) & =\int_{0}^{q_{1}} \partial_{i} C\left(t, \ldots, \gamma_{i}^{\varphi}(t, q), \ldots\right) d \gamma_{i}^{\varphi}(t, q), i \in N, i \neq 1 .
\end{aligned}
$$

Equalities (6) hold for any cost function $C$. Consider the function $C(x)=x_{1} \cdot x_{i}$ for some $i \in N, i \neq 1$. For this function the first equality (6) is written as

$$
\varphi_{1}(C, q)=\int_{0}^{q_{1}} \gamma_{i}^{\varphi}(t, q) d t .
$$

Consider now a coordinal transformation $\left\langle C^{f}, f^{-1}(q)\right\rangle$ of $\langle C, q\rangle$. For it equality (7) looks as:

$$
\varphi_{1}\left(N, C^{f}, f^{-1}(q)\right)=\int_{0}^{f^{-1}\left(q_{1}\right)} f\left(\gamma_{i}^{\varphi}\left(t, f^{-1}(q)\right) f^{\prime}(t) d t=\varphi_{1}(N, C, q) .\right.
$$

By COORD equalities (8) hold for any increasing function $f$ including continuously differentiable functions $f$.

Let such a function satisfy also the equalities $f\left(q_{i}\right)=q_{i}, f\left(q_{1}\right)=q_{1}$. Then from (8) it follows

$$
\begin{aligned}
\varphi_{1}\left(N, C^{f}, f^{-1}(q)\right) & =\int_{0}^{q_{1}} f\left(\gamma_{i}^{\varphi}(t, q)\right) d f(t) \\
& =\int_{0}^{q_{1}} f\left(\gamma_{i}^{\varphi}\left(f^{-1}(z), q\right) d z=\int_{0}^{q_{1}} \gamma_{i}^{\varphi}(t, q) d t .\right.
\end{aligned}
$$

The last equality means that for every strictly increasing function $f$ such that $f(0)=0$, $f\left(q_{i}\right)=q_{i}$ and every fixed $q>0$ the function $\gamma_{i}^{\varphi}$ as a function of $t$ satisfies the functional equation

$$
f \circ \gamma_{i}^{\varphi}=\gamma_{i}^{\varphi} \circ f .
$$

This can happen only if $\gamma_{i}^{\varphi}(t, q) \equiv t$ for all $q>0$.

Thus, the assumption that the path $\gamma^{\varphi}$ is strictly increasing leads to $\gamma_{1}^{\varphi}(t)=\gamma_{i}^{\varphi}(t)=$ $t, t \in\left[0, q_{1}\right]$ for all $i \in N, i \neq 1$, implying $q_{1}=q_{i} \forall i \in N$, and for this case the method $\varphi$ coincides both with the Aumann-Shapley method and with the serial method. 
Case 2. $\gamma_{i}^{\varphi}(t, q), i \in S \subset N$ are strictly increasing on an interval $t \in\left[\tau_{1}(q), \tau_{2}(q)\right]$. Let $1 \in S$. Then the inverse function $\left(\gamma_{1}\right)^{\varphi^{-1}}(z, q)$ is well-defined for $z \in\left[z_{1}, z_{2}\right]$, where $z_{1}=\gamma_{1}^{\varphi}\left(\tau_{1}(q), q\right), z_{2}=\gamma_{1}^{\varphi}\left(\tau_{2}(q), q\right)$.

Consider a cost function $C^{i} \in \mathfrak{C}, i \in S \backslash\{1\}$ such that $\partial_{i} C^{i}(x)=0$ for all $x_{1} \in$ $\left[0, q_{1}\right] \backslash\left[z_{1}, z_{2}\right]$ and $C^{i}(x)=\left(\gamma_{1}^{\varphi}\right)^{-1}\left(x_{1}, q\right) \cdot x_{i}$ for all $x_{i}$ and $x_{1} \in\left[z_{1}, z_{2}\right]$. Further in the proof we write for simplicity $\gamma$ instead of $\gamma^{\varphi}$. Then

$$
\varphi_{i}\left(N, C^{i}, q\right)=\int_{\tau_{1}(q)}^{\tau_{2}(q)} t d \gamma_{i}(t, q) .
$$

By COORD $\varphi\left(C^{i}, q\right)=\varphi\left(C^{i f}, f^{-1}(q)\right)$ for all increasing functions $f:\left[0, \max _{j} q_{j}\right] \rightarrow \mathbb{R}$ including those for which

$$
f\left(\tau_{k}(q)\right)=\tau_{k}(q), k=1,2 ; \quad f_{j}\left(q_{j}\right)=q_{j} \forall j \in N .
$$

For such functions

$$
\varphi_{i}\left(N, C^{i f}, f(q)\right)=\varphi_{i}\left(N, C^{i f}, q\right)=\int_{\tau_{1}(q)}^{\tau_{2}(q)} \gamma_{1}^{-1}\left(f\left(\gamma_{i}(t, q)\right), q\right) f^{\prime}\left(\gamma_{i}(t, q)\right) d \gamma_{i}(t, q)
$$

Introducing the new variable $\tau=\gamma_{i}^{-1}\left(f\left(\gamma_{i}(t, q), q\right)\right.$ we obtain $\gamma_{i}(\tau, q)=f\left(\gamma_{i}(t, q)\right)$ and the integral in (11) becomes equal to

$$
\int_{\tau_{1}(q)}^{\tau_{2}(q)} \gamma_{1}^{-1}\left(\gamma_{i}(\tau, q), q\right) d \gamma_{i}(\tau, q)
$$

By COORD the integrals in (9) and (12) are equal. Moreover, we can consider analogous functions $C^{i}$, increasing in $x_{1}$ only on subintervals $\left[z_{1}^{\prime}, z_{2}^{\prime}\right] \subset\left[z_{1}, z_{2}\right]$. Then we obtain that integrals in (9) and (12) are equal for any limits contained in $\left[\tau_{1}(q), \tau_{2}(q)\right]$. This fact implies $\gamma_{1}(t, q) \equiv \gamma_{i}(t, q)$ for $t \in\left[\tau_{1}(q), \tau_{2}(q)\right]$.

Therefore, we have proved that in each point $t \in[0, T]$ all increasing coordinates $\gamma_{i}(t, q)$ of the path $\gamma$ are equally increasing.

We shall call extreme methods from the set $\mathfrak{F}(A D D, D U M, C O O R D)$ compositions of incremental and serial methods, because by Lemma 1 every piece of the path corresponding to such a method is either an edge of the rectangle $[0, q]$, or the path corresponding to the serial method on some "sub-rectangle" $\left[\left(q_{S_{1}}, 0_{N \backslash S_{1}}\right),\left(q_{S_{1}}, q_{S_{2}}, 0_{N \backslash\left(S_{1} \cup S_{2}\right)}\right)\right]$ with $S_{1} \cap S_{2}=\emptyset$.

Consider the partition of $\mathbb{R}_{+}^{N}=\bigcup_{\alpha} O_{\alpha}$ into the "orthants" containing the vectors having the same ordering of coordinates:

$$
x, y \in O_{\alpha} \Longleftrightarrow \begin{aligned}
& x_{i}<x_{j} \leftrightarrow y_{i}<y_{j} \\
& x_{i}=x_{j} \leftrightarrow y_{i}=y_{j}
\end{aligned}
$$

Then for each common ordinal transformation $f=(f, \ldots, f)$

$$
x \in O_{\alpha} \Longrightarrow f(x)=\left(f\left(x_{1}\right), \ldots, f\left(x_{n}\right)\right) \in O_{\alpha} .
$$

Therefore, in order to describe the set $\operatorname{ext} \mathfrak{F}(A D D, D U M, C O O R D)$ it suffices to describe the sets of corresponding paths for demands belonging to orthants with no equal coordinates, with two equal coordinates, etc, with $n-1$ equal coordinates. Then 
any independent combination of such paths for all orthants will completely define the common ordinal path generated method. Formally we obtain the next result:

LEMMA 2. A method $\varphi$ is a path-generated method from the set $\mathfrak{F}(A D D, D U M$, COORD) if and only if for all demands $q \in O_{\alpha}$ it is the same composition of the incremental and serial methods, and different compositions may correspond to different orthants.

Proof. The 'only if' part follows from Lemma 1.

Let $\varphi$ be a path-generated method whose path $\gamma(t, q)$ from 0 to the demand vector $q$ satisfies the conditions of the lemma. Let $q \in O_{\alpha} \subset \mathbb{R}_{+}^{N}$ for some orthant $O_{\alpha}$. Then the path $\gamma(t, q)$ is piecewise linear, and consists of the pieces being either parts of the edges of the rectangle $[0, q]$, or intervals such that the coordinates $i \in S$ for some $S \subset N$ are equally increasing and others are constant. By the assumption of the lemma the paths $\gamma(t, q)$ for all $q \in O_{\alpha}$ consist of the same pieces.

Then the collection of the paths $\gamma(t, q)$ for demands belonging to each orthant completely define a path-generated method satisfying COORD. Since any path-generated method belongs to the set ext $\mathfrak{F}(A D D, D U M)$, the proof is complete.

Add the symmetry axiom to the conditions of Lemma 1 . Let $\varphi \in \operatorname{ext} \mathfrak{F}(A D D, D U M$, $S Y M, C O O R D)$ be an arbitrary method. The path determining the method does not depend on the cost function, hence, by symmetry, for demands with equal coordinates for each problem it is the diagonal of the corresponding rectangle $[0, q]$.

In general, the methods from the set ext $\mathfrak{F}(A D D, D U M, S Y M, C O O R D)$ are not demand continuous (e.g. the symmetric extension of incremental methods, which prescribe equal average awards to agents with equal demands). The next theorem shows that the serial method is the unique symmetric and demand continuous method in the set $\mathfrak{F}(A D D, D U M, S Y M, C O O R D)$.

THEOREM 4. The serial method is the only path generated method from $\mathfrak{F}(A D D, D U M$, $S Y M, C O O R D, D C O N T)$.

Proof. It is clear that the serial method verifies all the properties stated in the theorem.

Let now $\varphi$ be an arbitrary path-generated method, satisfying ADD, DUM, SYM, COORD, and DCON and not coinciding with the serial method. Let $N$ be fixed and $\gamma(t, q), t \in[0, T]$ be the path corresponding to the method $\varphi$, where $\max _{j \in N} q_{j} \leq T \leq$ $\sum_{j \in N} q_{j}$ (for any incremental method $T=\sum_{j \in N} q_{j}$, and for the serial method $T=$ $\max _{j \in N} q_{j}$.)

Let a sequence $q^{m}$ belong to the same orthant as $q$, and $q_{i}^{m} \rightarrow a$ when $m \rightarrow \infty$ for all $i \in N$. Then by Lemma 2 the paths $\gamma^{\varphi}\left(t, q^{m}\right)$ consist of the same pieces and by DCONT they should tend to the diagonal of the cube $[0, a]^{N}$, because of the symmetry of the method $\varphi$. However, since the method $\varphi$ is different from the serial one, the path $\gamma^{\varphi}$ either contains an edge of the rectangle $[0, q]$, and in this case it cannot tend to the diagonal, or there exists a partition $\left(S_{1}, \ldots, S_{k}\right), k \geq 2$ of $N$ such that the path $\gamma^{\varphi}(t, q)$ is generated by the serial method on the "sub-rectangles" $\left[0,\left(q_{S_{1}}, 0_{N \backslash S_{1}}\right)\right]$, $\left[\left(q_{S_{1}}, 0_{N \backslash S_{1}}\right),\left(q_{S_{1} \cup S_{2}}\right),\left(0_{N \backslash\left(S_{1} \cup S_{2}\right)}\right)\right], \ldots,\left[\left(q_{\cup_{i=1}^{k-1} S_{i}}, 0_{S_{k}}\right), q\right]$. If $q^{m} \rightarrow(a, \ldots, a)$, then the limiting path $\lim _{m \rightarrow \infty} \gamma^{\varphi}\left(t, q^{m}\right)$ would consist of the diagonals of the facets of the cube 
$[0, a]^{N}$, corresponding to the partition $\left(S_{1}, \ldots, S_{k}\right)$. Therefore, in this case the method $\varphi$ would not satisfy DCONT as well.

Theorem 4 gives an axiomatization of the serial method only on the set of pathgenerated methods. In order to obtain a genuine axiomatic characterization it is necessary to single out extreme points of the set $\mathfrak{F}(A D D, D U M, S Y M, C O O R D, D C O N T)$.

We give one more Decomposition axiom² implying the property "to be an extreme point" among the set $\mathfrak{F}(A D D, D U M)$ of cost sharing methods.

Decomposition (DECO). A method $\varphi$ satisfies the decomposition property if for every problem $\langle N, C, q\rangle$ for each $i \in N$ and $z_{i} \in\left(0, q_{i}\right)$ there exist $z_{j} \in\left[0, q_{j}\right], j \in N \backslash\{i\}$ such that for any $k \in N$

$$
\varphi_{k}(N, C, q)=\varphi_{k}(N, C, z)+\varphi_{k}\left(N, C^{z}, q-z\right),
$$

where $z=\left(z_{i}\right)_{i \in N}, C^{z}(x)=C(z+x), x \in[0, q-z]$.

LEMMA 3.

$$
\varphi \in \mathfrak{F}(A D D, D U M, D E C O) \Longrightarrow \varphi \in \operatorname{ext} \mathfrak{F}(\mathrm{ADD}, \mathrm{DUM}) .
$$

Proof. Let $\varphi \in \mathfrak{F}(A D D, D U M, D E C O)$. Then by [3] for every $q \in \mathbb{R}_{++}^{N}$ there exists a measure $\nu$ on the set $\Gamma(q)$ of all paths $\gamma(t, q)$ from 0 to $q$ such that

$$
\varphi_{i}(N, C, q)=\int_{\Gamma(q)} \varphi^{\gamma}(N, C, q) d \nu(\gamma),
$$

where $\varphi^{\gamma}$ is the path-generated method for the path $\gamma$.

We can apply Decomposition axiom repeatedly. Then we obtain that for each $i \in N$ and partition $\left[0, t_{1}^{i}, \ldots, t_{m}^{i}, q_{i}\right]$ of the interval $\left[0, q_{i}\right]$ there are partitions $\left[0, t_{1}^{j}\left(t_{1}^{i}\right), \ldots\right.$, $\left.t_{m}^{j}\left(t_{m}^{i}\right), q_{j}\right]$ of the intervals $\left[0, q_{j}\right]$ for each $j \in N \backslash\{i\}$ such that the support of the measure $\nu$ is contained in the set of paths going through the set

$$
\bigcup_{j=0}^{m}\left[\tau_{i}^{j}, \tau_{i}^{j+1}\right]
$$

where

$$
\left(\tau_{i}^{j}\right)_{k}= \begin{cases}t_{i}^{k}, & \text { if } j=i, \\ t_{j}^{k}\left(t_{i}^{k}\right) & \text { for } j \neq i,\end{cases}
$$

where $t_{j}^{m+1}=q_{j}, j \in N \backslash\{i\}$.

The set (15) is the union of a finite set of rectangles $\left[\tau_{i}^{k}, \tau_{i}^{k+1}\right]$ which may intersect only in one vertex and, hence, all paths through the set (15) pass through the points $0, \tau_{i}^{1}, \ldots, \tau_{i}^{m}, q$, where $\tau_{i}^{k+1} \geq \tau_{i}^{k}, k=1, \ldots, m$.

Let the partition $\left(0, t_{1}^{i}, \ldots, t_{m}^{i}, q\right)$ become smaller as $m \rightarrow \infty$ such that the intervals between adjacent points of the corresponding partitions $\left(0 . t_{1}^{i}, \ldots, t_{m}^{i}, q_{i}\right)$ tend to zero: $t_{k+1}^{i}-t_{k}^{i} \rightarrow_{m \rightarrow \infty} 0$.

\footnotetext{
${ }^{2}$ This axiom under the name "Self Consistency" was firstly introduced by M. Koster [5] who applied it to the characterization of the Sprumont ordinal serial rule [8].
} 
Then the set of paths $\Gamma(q)$ in definition (14) tightens to a single path $\gamma$ formed by the limit as $m \rightarrow \infty$ of the sequence $\left(0, \tau_{i}^{1}, \ldots, \tau_{i}^{m}, q\right)$, and formula (14) implies

$$
\varphi(N, C, q)=\varphi^{\gamma}(N, C, q)
$$

Theorem 4 and Lemma 3 imply the following axiomatization of the serial method:

THEOREM 5. The serial method is the only method satisfying ADD, DUM, SYM, COORD, DCONT, and DECO.

Proof. To prove that the serial method $s$ satisfies all the axioms it suffices only to show that it satisfies DECO. Let $i \in N$ and $z_{i}=t \in\left(0, \max _{i} q_{i}\right)$. For $j \in N \backslash\{i\}$ define the vector $z$ by

$$
z_{j}= \begin{cases}z_{i}, & \text { if } t<q_{j}, \\ q_{j}, & \text { if } t \geq q_{j} .\end{cases}
$$

Therefore, for $j \in N$ such that $z_{j} \geq q_{j}$

$$
s_{j}(N, C, q)=s_{j}(N, C, z) .
$$

If $z_{j}<q_{j}$ then

$$
\begin{aligned}
s_{j}(N, C, q) & =\int_{0}^{t} \partial C_{j}((t e) \wedge q) d t+\int_{t}^{q_{j}} \partial_{j} C((t e) \wedge q) d t \\
& =s_{j}(N, C, z)+s_{j}\left(N, C^{t}, q-z\right) .
\end{aligned}
$$

Let now $\varphi$ be an arbitrary method satisfying all the axioms. By Lemma 3 it is path generated. By Theorem 4 it is the serial method.

The following examples of methods satisfying all the axioms stated in the Theorem except for any one, prove independence of the axioms.

Without ADD. The Moulin-Shenker serial method [7].

This method $s^{M S h}$ is defined only for the class $\mathfrak{C}_{1} \subset \mathfrak{C}$ of cost functions $C$, whose partial derivatives are bounded:

$$
C \in \mathfrak{C}_{1} \Longrightarrow 0<a(C) \leq \partial_{i} C \leq b(C)
$$

for some numbers $a(C), b(C)$. For each problem $\langle N, C, q\rangle$ it is generated by the path $\gamma^{M S h}(t, q)$ such that the incremental costs generated by a small move along the path is shared equally among the agents not yet fully served:

$$
\begin{aligned}
s_{i}^{M S h}(N, C, q) & =\int_{0}^{\infty} \partial C_{i}\left(\gamma^{M S h}(t, q)\right) d \gamma_{i}(t, q) \\
& =\frac{C\left(\gamma^{M S h}\left(t_{i}, q\right)\right.}{n-i+1}-\sum_{j<i} \frac{C\left(\gamma^{M S h}\left(t_{j}, q\right)\right.}{(n-j+1)(n-j)} \forall j<i, i \in N,
\end{aligned}
$$

where $q_{1} \leq q_{2} \leq \ldots \leq q_{n}, t_{i}=\inf \left\{t \mid \gamma_{i}(t, q)=q_{i}\right\}$, and the path $\gamma^{M S h}(t, q)$ is determined from differential equations

$$
\frac{d \gamma_{i}(t, q)}{d t}=\left\{\begin{array}{ll}
\frac{1}{\partial_{i} C(\gamma(t, q))} & \text { if } \gamma_{i}(t, q)<q_{i}, \\
0 & \text { otherwise, }
\end{array} \quad \forall i \in N\right.
$$


It is not difficult to extend the method for all cost functions $C \in \mathfrak{C}$ by approximations $C_{m} \rightarrow C, C_{m} \in \mathfrak{C}_{1}$. The definition of the path $\gamma^{M S h}$ implies that the Moulin-Shenker method satisfies Ordinality (and, all the more, Coordinality), Dummy, Symmetry, Demand Continuity, and Decomposition.

Without DUM. The direct serial method [7]

$$
s_{i}^{d}(N, C, q)=\frac{C(q(i))}{n-i+1}-\sum_{j<i} \frac{C(q(j))}{(n-j+1)(n-j))} \forall i \in N,
$$

where $q_{j}(i)=\min \left\{q_{i}, q_{j}\right\}$. This method is defined by the same path as the serial method, however, the agents' shares are not the sums of integrals of their incremental costs, but the sum of average total increments. It is clear that the direct serial method satisfies all the axioms except for Dummy.

Without COORD. The Aumann-Shapley method determined by

$$
\left.x_{i} N, C, q\right)=\int_{0}^{q_{i}} \partial_{i} C\left(\frac{t}{q_{i}} \cdot q\right) d t, i \in N,
$$

does not satisfy Coordinality, but evidently, satisfies all other axioms.

Without SYM. The incremental methods. Each incremental method is determined by a permutation $\pi: N \rightarrow N$ of agents, and, hence, it is not symmetric. However, all other axioms are satisfied.

Without DCONT. The anonymous compositions $\varphi^{a}$ of incremental and serial methods.

Let $N$ be fixed and $\varphi^{\pi}$ be the incremental method corresponding to the permutation $\pi: N \rightarrow N$. Define the method $\varphi^{a}$ as follows: let $\langle N, C, q\rangle$ be an arbitrary problem, $q \in O_{\pi}$, i.e. the demand vector $q$ has distinct coordinates ordered in accordance with the permutation $\pi$. Let

$$
\varphi_{i}^{a}(N, C, q)=\varphi_{i}^{\pi}(N, C, q) \forall i \in N .
$$

Let now $q^{\prime}$ be an arbitrary demand vector with distinct coordinates. Then for some permutation $\tau: N \rightarrow N \tau q^{\prime} \in O_{\pi}$. Put

$$
\varphi_{i}^{a}\left(N, C, q^{\prime}\right)=\varphi_{i}^{\tau^{-1}}\left(N, C, \tau q^{\prime}\right) .
$$

It remains to define the method $\varphi^{a}$ for demand vectors possessing equal coordinates. Define the path for demands $q$ where

$$
q_{\pi 1} \leq q_{\pi 2} \leq \ldots \leq q_{\pi n}
$$

If $q_{\pi 1}<q_{\pi 2}$, then link $\mathbf{0}$ to $\left(q_{\pi 1}, \mathbf{0}\right)$. if $q_{\pi 1}=q_{\pi 2}$, then link $\mathbf{0}$ to $\left(q_{\pi 1}, q_{\pi 2}, \ldots, q_{\pi k}, \mathbf{0}\right)$, where $q_{\pi 1}=q_{\pi 2}=\ldots=q_{\pi k}<q_{\pi(k+1)}$. Then repeat the procedure till we come to the vector $q$. Since other demand vectors with equal coordinates are some permutations of vectors from $O_{\pi}$, we can define the corresponding paths for them as the corresponding permutations of the paths defined above. Thus, the paths to all vectors $q \in \mathbb{R}_{+}^{N}$ have been determined, and the method $\varphi^{a}$ is generated by these paths.

It is clear that the method $\varphi^{a}$ satisfies ADD, DUM, SYM, COORD. Since the paths generating the method for each $q$ belong to the closure of the unique orthant $O_{\pi}$, the method satisfies DECO. 
Without DECO. The Shapley-Shubik method. This method is not path-generated, hence it does not satisfy Decomposition. Clearly, it satisfies all other axioms.

\section{References}

[1] L. Billera and D. Health, Allocation of shared cost: A set of axioms yielding a unique procedure, Math. Oper. Res. 7 (1992), 32-39.

[2] E. Friedman, Paths and consistency in additive cost sharing, International Journal of Game Theory 32 (2003), 501-518.

[3] E. Friedman and H. Moulin, Three methods to share joint costs or surplus, J. Econ. Theory 87 (1999), 275-312.

[4] O. Haimanko, Partially symmetric values, mimeo, Hebrew University, Jerusalem, 1998.

[5] M. A. L. Koster, Cost Sharing in Production and Network Exploitation, CentER Dissertation Series, no 58, Tilburg University, 1999.

[6] H. Moulin, Axiomatic cost and surplus sharing methods, in: Handbook on Social Choice and Welfare, K. J. Arrow, A. K. Sen, and K. Suzumura (eds.), Elsevier Science, 2002, 289-357.

[7] H. Moulin and S. Shenker, Distributive and additive costsharing of an homogeneous good, Games Econ. Behav. 27 (1999), 299-330.

[8] Y. Sprumont, Ordinal cost sharing, Journal of Economic Theory 81 (1998), 126-162.

[9] Y. Sprumont and Y. Wang, Ordinal additive cost sharing methods must be random order values, mimeo, Université de Montréal, 1996.

[10] W. Thomson, Axiomatic and game-theoretic analysis of bankruptcy and taxation problems: a survey, Mathematical Social Sciences 45 (2003), 249-297. 